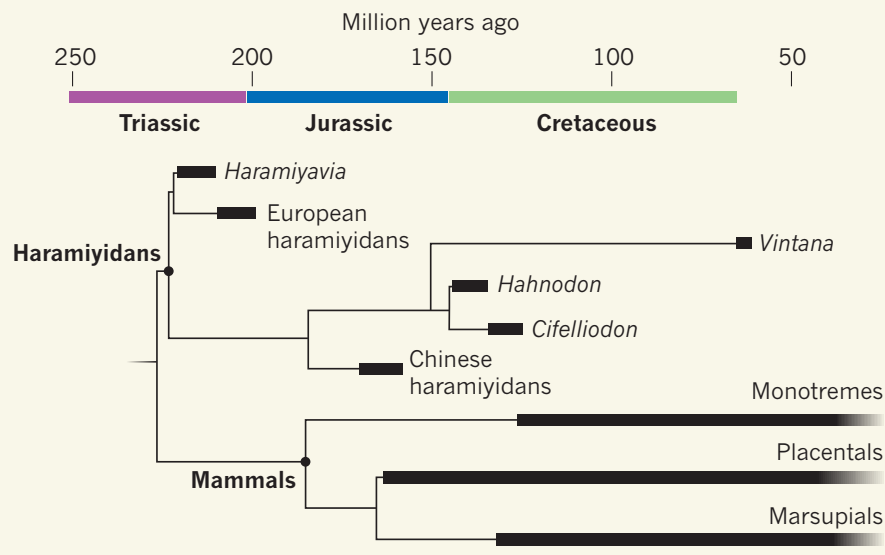

b

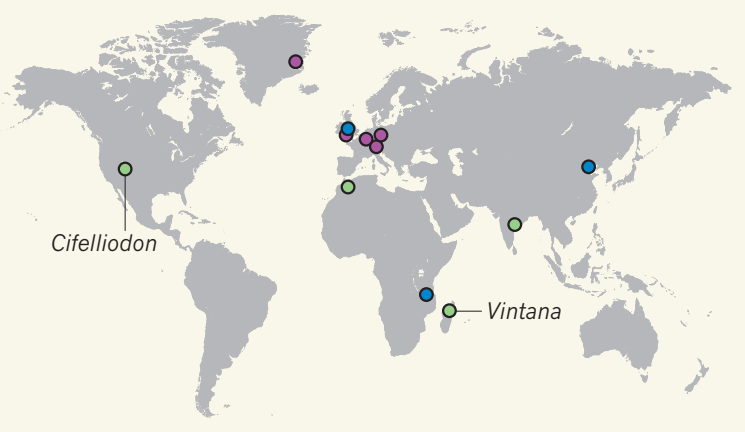

Figure 2 Re-evaluating the evolution and biogeography of haramiyidans. a, Huttenlocker $\mathrm{et} \mathrm{al.}{ }^{1}$ analysed relationships between the early branches of the family tree for mammals and their more primitive relatives. The resulting evolutionary tree indicates that haramiyidans are not mammals, contrary to some previous evidence ${ }^{5,6,8,9}$. The analysis also places the Cretaceous genus Vintana in Haramiyida for the first time. b, Cretaceous haramiyidans (indicated by green circles) have previously been found in northern Africa and possibly
India. The authors' analysis expands the Cretaceous range of haramiyidans to Madagascar (Vintana) and North America (Cifelliodon). Combined with the fact that other fossils of haramiyidans from the Triassic (purple) have been found in Europe and Greenland, and that haramiyidans from the Jurassic (blue) have been found in Europe, China and Tanzania, this work implies a much broader temporal and geographical distribution of haramiyidans than had previously been hypothesized. that, although the Chinese haramiyidans are represented by complete skeletons, the specimens are essentially 2D. Most of the skulls are little more than flattened outlines, which limits their usefulness for informing mammalian relationships.

Cifelliodon is one of the first skulls preserved in three dimensions from the haramiyidan lineage. As such, it is a crucial piece of the evolutionary puzzle. Huttenlocker and colleagues' phylogeny puts Haramiyida (and so Cifelliodon) outside Mammalia (Fig. 2a). Thus, their work favours a model in which early mammals diversified rapidly during the Jurassic.

Finally, Huttenlocker et al. provide evidence that Cifelliodon is closely related to Cretaceous species from northern Africa (Hahnodon taqueti) and Madagascar (Vintana sertichi), the latter of which had not previously been assigned to Haramiyida. This implies a much broader temporal and geographical distribution for Haramiyida than has been assumed (Fig. 2b), indicating the need to reassess the biogeographical history of the group. The authors conclude that haramiyidans had a global distribution during the JurassicCretaceous transition, and that land bridges aiding vertebrate dispersal existed long after the fragmentation of the supercontinent Pangaea - much later than previously recognized. An alternative hypothesis that is perhaps more consistent with current palaeogeographical models ${ }^{11}$ is that haramiyidans, like many vertebrate groups, had a Pangaean distribution in the Jurassic period and evolved in isolation thereafter, as landmasses separated during the Cretaceous period. The best way to test these competing hypotheses is with the discovery of more well-preserved fossils, like this exquisite skull of Cifelliodon.
Simone Hoffmann is in the Department of Anatomy, New York Institute of Technology, College of Osteopathic Medicine, Old Westbury, New York 11568, USA. David W. Krause is in the Department of Earth Sciences, Denver Museum of Nature \& Science, Denver, Colorado 80205, USA. e-mails:shoffm04@nyit.edu; david.krause@dmns.org

1. Huttenlocker, A. K., Grossnickle, D. M., Kirkland, J. I., Schultz, J. A. \& Luo, Z.-X. Nature 558, 108-112 (2018).

2. Kielan-Jaworowska, Z., Cifelli, R. L. \& Luo, Z.-X. Mammals from the Age of Dinosaurs: Origins, Evolution, and Structure (Columbia Univ. Press, 2004).
3. Rowe, T. B., Macrini, T. E. \& Luo, Z.-X. Science 332 955-957 (2011).

4. Koyabu, D., Maier, W. \& Sánchez-Villagra, M. R. Proc. Natl Acad. Sci. USA 109, 14075-14080 (2012).

5. Krause, D. W. et al. Nature 515, 512-517 (2014)

6. Zheng, X., Bi, S., Wang, X. \& Meng, J. Nature 500, 199-202 (2013).

7. Zhou, C.-F., Wu, S., Martin, T. \& Luo, Z.-X. Nature 500, 163-167 (2013).

8. Bi, S., Wang, Y., Guan, J., Sheng, X. \& Meng, J. Nature 514, 579-584 (2014).

9. Han, G. et al. Nature 551, 451-456 (2017).

10.Luo, Z.-X. et al. Nature 548, 326-329 (2017).

11.Torsvik, T. H. \& Cocks, R. M. Earth History and

Palaeogeography (Cambridge Univ. Press, 2017).

\title{
ASTRONOMY
}

\section{A fresh approach to stellar benchmarking}

\begin{abstract}
An avalanche of data is about to revolutionize astronomy, but the options for validating those data have been limited. High-precision measurements from the Hubble Space Telescope enable a much-needed alternative option.
\end{abstract}

\section{RACHAEL BEATON}

1 ry this experiment: extend your thumb at arm's length and close one eye at a time. Your thumb will seem to 'jump' between two positions as you switch the eye that is closed. That jump is known as parallax. If you measure the jump as well as the distance between your eyes, you can use trigonometry to calculate the distance to your thumb. Astronomers use parallax, on a much greater scale, to measure distances to astronomical objects. Writing in The Astrophysical Journal Letters, Brown et al. ${ }^{1}$ report that they have achieved this for the nearby star cluster NGC 6397, using the Hubble Space Telescope. Their method will provide a crucial means of validating the wealth of parallax data released this year from the European Space Agency's Gaia mission ${ }^{2}$.

It is a challenge to find a topic in astronomy that does not rely on the astronomical distance 


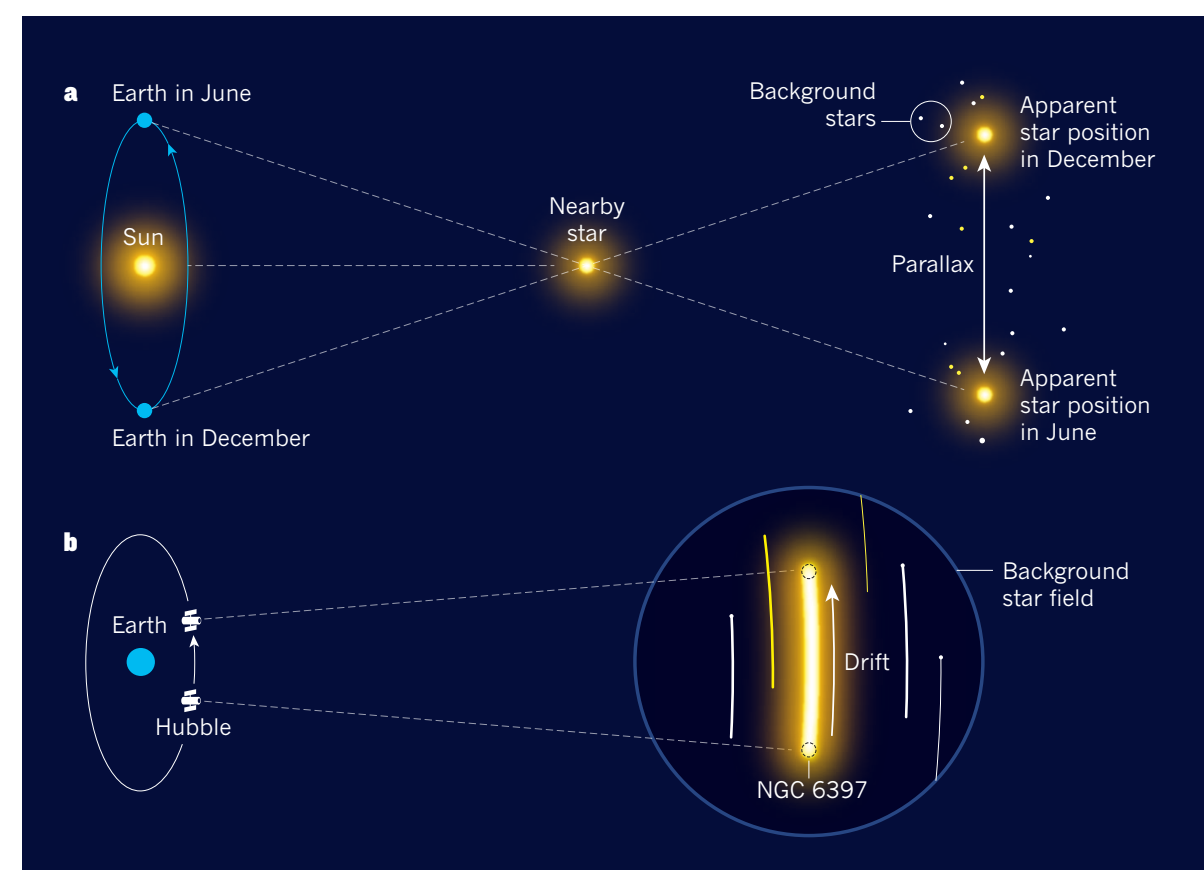

Figure 1 | Using parallax to measure astronomical distances. Objects viewed along two lines of sight have different apparent positions relative to their background, and the distance between those positions is known as the parallax. a, Distances to a nearby star are determined by measuring the parallax between two images of the star that were taken six months apart. Because the distance between the two observation positions is known to be the diameter of Earth's orbit around the Sun, the distance to the star can be calculated using trigonometry. $\mathbf{b}$, Brown et al. ${ }^{1}$ have measured the distance to the nearby star cluster NGC 6397. A camera on the Hubble Space Telescope took two long exposures six months apart, so that the cluster was visible as a 'streak' that results from the telescope's orbital motion around Earth (the apparent drift of the cluster has been exaggerated, for clarity). Each position along the streak provides a different measurement of the position of each star in the cluster, thereby allowing the apparent positions of the stars to be measured more precisely than from 'snapshot' images. These measurements enabled the distance to the cluster to be determined.

scale - a collection of methods applied in series to determine distances that are too large to be measured directly. Distances are used as conversion factors for deriving the physical quantities of celestial objects from observations and, therefore, they are essential for constructing models of the Universe. The foundations of the astronomical distance scale are trigonometric parallaxes for individual stars. These parallaxes enable us to calibrate the physical properties of those stars, which can then be used to infer properties of ever more distant stars, star clusters and galaxies. On the largest distance scales, they can even be used to calculate the size of the Universe.

A stellar parallax was first measured in 1838 by the astronomer Friedrich Bessel ${ }^{3}$. Many more have been recorded since. Until earlier this year, about 2 million reliable measurements ${ }^{4}$ had been made. This sounds like an impressive number, but effectively spanned only the astronomical cul-de-sac in which the Sun resides. That number increased to roughly 1 billion following the release in April of data from Gaia ${ }^{2}$, which surveyed a region well beyond the Solar System, almost halfway across the Galaxy. Until the publication of Brown and colleagues' data, only one technique - very-long-baseline interferometry ${ }^{5}$ - was capable of measuring parallax directly on such distance scales. This was a concern because astronomers worldwide are poised to use the Gaia data in their research, and so it would be desirable to have more than one direct method for measuring stellar parallaxes to help validate the Gaia data.

The basic experimental set-up for measuring stellar parallax is identical to that described for observing your thumb. First, two images of the same astronomical object are taken with an interval of six months (Fig. 1a). This ensures that they are captured at positions separated by the diameter of Earth's orbit around the Sun, in the same way that observing your thumb from each eye provides two viewpoints that are separated by a known distance. Second, the apparent displacement of the target star is determined. This involves measuring the position of the star in each image with high precision, and then measuring that position in relation to a set of reference objects (stars or galaxies) from the same image. Both tasks are conceptually simple yet tricky to achieve in practice. Brown et al. address them in interesting ways.

To measure the position of their target star cluster, Brown and colleagues took the two images with a camera on the Hubble Space Telescope using a long exposure, so that the cluster's stars 'drift' across the images as a result of the telescope's orbital motion around Earth
(Fig. 1b). This technique, known as spatial scanning ${ }^{6,7}$, produces images of the target as a 'streak'. Every position along the streak provides a different measurement of the position of each star in the cluster.

The images of NGC 6397 taken by Brown et al. comprise more than 1,000 individual measurements, which increases the overall precision by more than 30 -fold, compared with a conventional 'snapshot'. Moreover, each measurement was made for numerous stars in that cluster. Spatial scanning has previously been used by researchers from the same group to study single stars, several thousand light years away, that are exceptionally bright ${ }^{6,7}$, but Brown et al. are the first to apply this technique to faint stars in a cluster at these sort of distances. (NGC 6397 is about 7,500 light years, or 2,390 parsecs, from Earth.)

The authors then used the same spatial scanning technique to measure the position of non-cluster stars in the background star field with incredible precision, enabling them to determine the displacement of cluster stars relative to each non-cluster star. But these relative parallaxes must be put into an absolute frame of reference, and setting such a frame is a complicated task. To do this, Brown and colleagues required coarse estimates (accurate to $\pm 15 \%$ of the true value) of the parallaxes for the non-cluster stars. The authors obtained these by determining the type and size of each star, and then assigning each the mean physical properties of its class, from which its distance (and therefore its parallax) can be determined ${ }^{8}$.

The Gaia mission also uses a scanning technique to obtain the positions of target objects, but sets the absolute frame using a sample of quasars (point-like galaxies that are unfathomably far away) from across the entire night sky ${ }^{9,10}$. Brown and colleagues' frame of reference has systematic uncertainties that are distinct from those of Gaia, and it could therefore provide a direct, independent means of testing the Gaia reference frame if it were to be expanded to include more star clusters.

The highly precise, long-distance parallax measurements provided by Gaia are a leap forward for astronomy. But, as in all fields of science, precision is not the only source of uncertainty. It is also crucial to understand the systematic uncertainty that is associated with a reference frame, partly so that this parameter can be included in data analyses, but also to devise a better means of establishing the frame. Systematic uncertainties can be reduced only by the addition of fresh, independent information, such as that provided by Brown and co-workers. The work involved in establishing these safeguards can be tedious and is often overlooked, but it is the bedrock of scientific progress.

Rachael Beaton is in the Department of Astrophysical Sciences, Princeton University, Princeton, New Jersey 08544, USA. 
e-mail: rbeaton@princeton.edu

1. Brown, T. M. et al. Astrophys. J. Lett. 856, L6 (2018)

2. Gaia Collaboration. Preprint at https://arXiv.org/ abs/1804.09365 (2018).

3. Bessel, F. W. Mon. Not. R. Astron. Soc. 4, 152-161 (1938)
4. Lindegren, L. et al. Astron. Astrophys. 595, A4 (2016).

5. Reid, M. J. \& Honma, M. Annu. Rev. Astron. Astrophys. 52, 339-372 (2014).

6. Riess, A. G., Casertano, S., Anderson, J., MacKenty, J. \& Filippenko, A. V. Astrophys. J. 785 161 (2014)
7. Casertano, S. et al. Astrophys. J. 825, 11 (2016)

8. Benedict, G. F et al. Publ. Astron. Soc. Pacif. 106 327-336 (1994).

9. Gaia Collaboration. Preprint at https://arXiv.org/ abs/1804.09377 (2018).

10.Gaia Collaboration. Astron. Astrophys. 595, A1 (2016).

\section{Human embryonic stem cells get organized}

An embryo's body plan is established by a structure called the organizer. Evidence of this structure in humans has been lacking, but a stem-cell-based protocol has now enabled researchers to demonstrate its existence. SEE LETTER P.132

\section{OLIVIER POURQUIÉ}

I 1924, Hilde Mangold and Hans Spemann performed what became one of the most famous experiments in developmental biology. They grafted various parts of one grafted region induced unpigmented cells from the host to form an extra embryo, resulting in a 'double embryo' reminiscent of conjoined twins ${ }^{1}$ (Fig. 1a). The duo named the grafted region the organizer, because of its extraordinary ability to organize the host cells around it. But in the almost 100 years since this experiment, technical and ethical difficulties have prevented researchers from demonstrating the presence of an organizer in human embryos. On page 132, Martyn et al. ${ }^{2}$ use stem cells to circumvent these challenges and provide the first experimental description of the human organizer.

To fully understand the importance of the organizer, we must go back to the earliest stages of embryonic development. In vertebrates, the fertilized egg rapidly divides to form a ball of poorly organized cells. At a particular developmental time point, some cells on the surface of this ball become internalized, forming tissues called the endoderm and the mesoderm, which respectively give rise to the gut and to muscles and the skeleton. Other cells remain external and give rise to the skin and the nervous system. This fundamental process of internalization is called gastrulation.

The organizer lies immediately adjacent to the site at which cells become internalized during gastrulation. It gives rise to specific tissues lying along the midline of the embryo, including the notochord - a structure that controls aspects of development of the central nervous system and eventually contributes to the intervertebral discs. An equivalent of the salamander organizer has been found in fish a pigmented salamander embryo onto an unpigmented host embryo, and showed that and birds and in mammals such as rodents ${ }^{3}$. In mammals, the structure that acts as an organizer is called the node because it resembles a knot, and the site of internalization is called the primitive streak.

Unlike salamander embryos, mammals develop in the mother's womb. Accessing and culturing mammalian embryos is therefore difficult. Indeed, it wasn't until 1994 that grafts of a mouse node into a host embryo provided experimental proof of the existence of a structure that has organizer properties in mammals ${ }^{4}$. Although no perfect second embryos were formed in these experiments, the grafted nodes did induce the formation of host-derived neural tissues and sometimes other embryonic tissues.

Human embryos greatly resemble mouse embryos and contain a structure that looks similar to the mouse node ${ }^{4}$. Theoretically, showing that this structure does indeed have the role of an organizer would require researchers to access embryos at three weeks of age (when gastrulation occurs), to graft the node onto a host embryo, and to test whether it induces the formation of a host-derived nervous system and skeletal structures. However, obtaining intact human embryos at this stage, for example from a pregnancy termination, is extremely problematic. Thus, whether the node represents a functional organizer in human embryos has remained unproven.

One alternative would be to let embryos obtained from in vitro fertilization (IVF) develop in culture until the three-week stage, when the node should be present. However, following an ethical consensus that is enshrined in law in many countries, human embryos cannot be cultured in vitro beyond 14 days, making these studies currently impossible.

A second alternative involves the use of pluripotent stem cells, which can give rise to all the body's cell lineages. Protocols to direct in vitro differentiation of these cells make it possible to recapitulate several aspects of

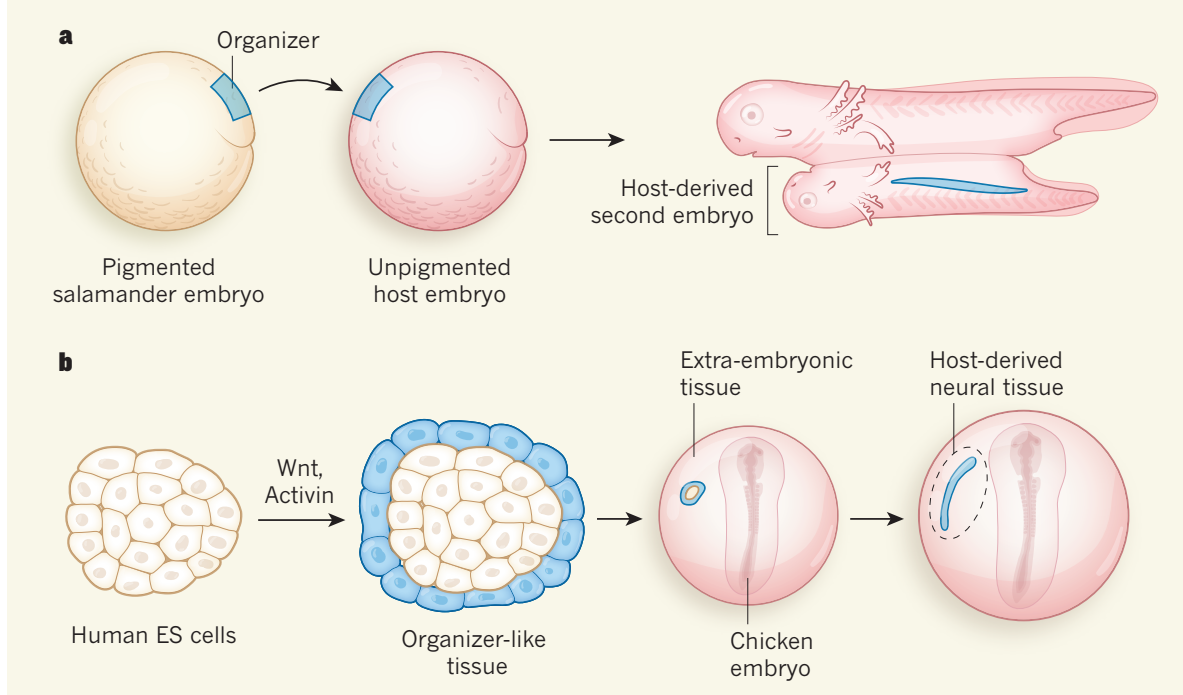

Figure 1 | Experimental demonstration of organizer structures. a, In 1924, an experiment ${ }^{1}$ revealed the properties of an embryonic structure called the organizer. When taken from a pigmented salamander embryo and grafted onto an unpigmented host, the organizer induced the formation of a second embryo derived from unpigmented host cells. b, Martyn et al. ${ }^{2}$ have demonstrated the existence of human cells endowed with similar properties, using human embryonic stem (ES) cells. The authors treated circular discs of ES cells with the growth-factor proteins Wnt and Activin to produce organizer-like cells (blue). When the discs are grafted onto the extra-embryonic tissue around a chick embryo, they induce the host tissue to form an elongated stretch of neural tissue - the standard test for organizer properties. 